\title{
Crystal structure of $(4 \mathrm{a} \alpha, 4 \mathrm{~b} \alpha, 10 \mathrm{a} \alpha, 10 \mathrm{~b} \alpha)-2,3,4 \mathrm{a}, 10 \mathrm{~b}$-tetrachloro- 1,4-dioxo-1,4,4a,4b,5,6,7,8,9,10,10a,10b-dodecahydro- benzo[3,4]cyclobuta[1,2]cyclooctane, $\left(\mathrm{C}_{8} \mathrm{H}_{14}\right)\left(\mathrm{C}_{6} \mathrm{Cl}_{4} \mathrm{O}_{2}\right)$
}

\author{
K. Peters ${ }^{*, I}$, E.-M. Peters ${ }^{I}$, M. Braun ${ }^{I I}$, St. Wolff ${ }^{\text {II }}$ and M. Christl ${ }^{\text {II }}$ \\ I Max-Planck-Institut für Festkörperforschung, Heisenbergstraße 1, D-70506 Stuttgart, Germany \\ II Universität Würzburg, Institut für Organische Chemie, Am Hubland, D-97074 Würzburg, Germany
}

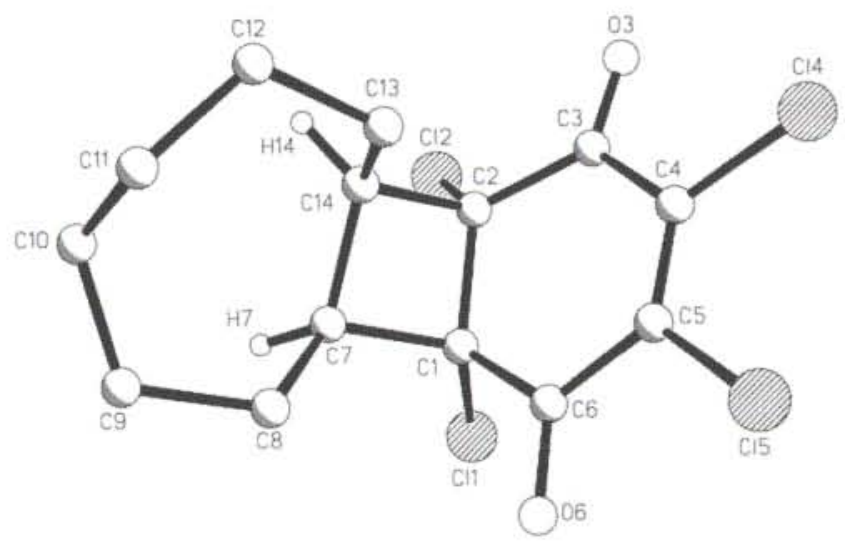

\begin{abstract}
$\mathrm{C}_{14} \mathrm{H}_{14} \mathrm{Cl}_{4} \mathrm{O}_{2}$, monoclinic, $P 12 / / n 1$ (No. 14), $a=12.717(2) \AA$, $b=9.2786(7) \dot{A}, c=12.843(8) \AA, \beta=94.789(7)^{\circ}, V=1510.1 \AA^{3}$, $Z=4, R_{\mathrm{gt}}(F)=0.059, w R(F)=0.063, T=293 \mathrm{~K}$.
\end{abstract}

\section{Source of material}

The title compound was prepared by irradiation of a solution of chloranil and cyclooctene in benzene at $293 \mathrm{~K}$ in a Rayonet photochemical reactor RPR-100 $(\lambda=350 \mathrm{~nm})[1,2]$.
Table 1. Data collection and handling.

$\begin{array}{ll}\text { Crystal: } & \text { colourless prism, size } 0.4 \times 0.5 \times 0.6 \mathrm{~mm} \\ \text { Wavelength: } & \text { Mo } K_{\alpha} \text { radiation }(0.71073 \mathrm{~A}) \\ \mu: & 7.80 \mathrm{~cm}^{-1} \\ \text { Diffractometer, scan mode: } & \text { Siemens P4, } 1 \\ 2 \theta_{\text {max }}: & 55^{\circ} \\ N(h k l)_{\text {measured }}, N(h k l)_{\text {lnique: }}: & 7293,3469 \\ \text { Criterion for } F_{\text {obs }}, N(h k l)_{\mathrm{gg}}: & F_{\text {obs }}>3 \sigma\left(F_{\text {obs }}\right), 3026 \\ N(\text { param })_{\text {refined: }} & 181 \\ \text { Program: } & \text { SHELXTL-plus [3] }\end{array}$

Table 2. Atomic coordinates and displacement parameters (in $\AA^{3}$ ).

\begin{tabular}{lllrll}
\hline Atom & Site & $x$ & $y$ & $z$ & \multicolumn{1}{l}{$U_{\text {iso }}$} \\
\hline $\mathrm{H}(7)$ & $4 e$ & $0.4758(3)$ & $0.1303(3)$ & $0.1292(2)$ & 0.08 \\
$\mathrm{H}(8 \mathrm{~A})$ & $4 e$ & $0.5966(3)$ & $-0.0259(3)$ & $0.1659(3)$ & 0.08 \\
$\mathrm{H}(8 \mathrm{~B})$ & $4 e$ & $0.5696(3)$ & $-0.0140(3)$ & $0.2825(3)$ & 0.08 \\
$\mathrm{H}(9 \mathrm{~A})$ & $4 e$ & $0.4285(4)$ & $-0.0989(4)$ & $0.1154(4)$ & 0.08 \\
$\mathrm{H}(9 \mathrm{~B})$ & $4 e$ & $0.4736(4)$ & $-0.1999(4)$ & $0.2062(4)$ & 0.08 \\
$\mathrm{H}(10 \mathrm{~A})$ & $4 e$ & $0.3025(4)$ & $-0.1482(5)$ & $0.2319(4)$ & 0.08 \\
$\mathrm{H}(10 \mathrm{~B})$ & $4 e$ & $0.3252(4)$ & $0.0174(5)$ & $0.2246(4)$ & 0.08 \\
$\mathrm{H}(11 \mathrm{~A})$ & $4 e$ & $0.3219(4)$ & $-0.1214(5)$ & $0.3958(3)$ & 0.08 \\
$\mathrm{H}(11 \mathrm{~B})$ & $4 e$ & $0.4431(4)$ & $-0.1073(5)$ & $0.3838(3)$ & 0.08 \\
$\mathrm{H}(12 \mathrm{~A})$ & $4 e$ & $0.3755(3)$ & $0.0777(4)$ & $0.4867(3)$ & 0.08 \\
$\mathrm{H}(12 \mathrm{~B})$ & $4 e$ & $0.3090(3)$ & $0.1328(4)$ & $0.3863(3)$ & 0.08 \\
$\mathrm{H}(13 \mathrm{~A})$ & $4 e$ & $0.4634(3)$ & $0.2703(3)$ & $0.4345(3)$ & 0.08 \\
$\mathrm{H}(13 \mathrm{~B})$ & $4 e$ & $0.5299(3)$ & $0.1377(3)$ & $0.4023(3)$ & 0.08 \\
$\mathrm{H}(14)$ & $4 e$ & $0.3818(2)$ & $0.2509(3)$ & $0.2711(2)$ & 0.08 \\
& & & & & \\
\hline
\end{tabular}

Table 3. Atomic coordinates and displacement parameters (in $\AA^{2}$ ).

\begin{tabular}{|c|c|c|c|c|c|c|c|c|c|c|}
\hline Atom & Site & $x$ & $y$ & $z$ & $U_{11}$ & $U_{22}$ & $U_{33}$ & $U_{12}$ & $U_{13}$ & $U_{23}$ \\
\hline $\mathrm{Cl}(1)$ & $4 e$ & $0.6523(1)$ & $0.3199(1)$ & $0.08102(8)$ & $0.128(1)$ & $0.0720(7)$ & $0.0625(6)$ & $-0.0175(6)$ & $0.0418(6)$ & $0.0006(5)$ \\
\hline $\mathrm{Cl}(2)$ & $4 e$ & $0.45853(9)$ & $0.48969(9)$ & $0.15844(8)$ & $0.0915(7)$ & $0.0441(5)$ & $0.0760(6)$ & $0.0021(4)$ & $-0.0215(5)$ & $0.0143(4)$ \\
\hline $\mathrm{Cl}(4)$ & $4 e$ & $0.69794(9)$ & $0.4902(1)$ & $0.52131(7)$ & $0.0867(7)$ & $0.0893(8)$ & $0.0592(6)$ & $0.0031(5)$ & $-0.0066(5)$ & $-0.0255(5)$ \\
\hline $\mathrm{Cl}(5)$ & $4 e$ & $0.82592(8)$ & $0.2242(1)$ & $0.44927(8)$ & $0.0603(5)$ & $0.0693(6)$ & $0.0784(6)$ & $0.0071(4)$ & $-0.0064(5)$ & $0.0154(5)$ \\
\hline$C(1)$ & $4 e$ & $0.6033(3)$ & $0.2705(3)$ & $0.2008(2)$ & $0.055(2)$ & $0.042(1)$ & $0.043(1)$ & $-0.004(1)$ & $0.009(1)$ & $-0.002(1)$ \\
\hline $\mathrm{C}(2)$ & $4 e$ & $0.5282(2)$ & $0.3766(3)$ & $0.2516(2)$ & $0.051(2)$ & $0.035(1)$ & $0.051(2)$ & $0.001(1)$ & $-0.001(1)$ & $0.004(1)$ \\
\hline$C(3)$ & $4 e$ & $0.5720(3)$ & $0.4668(3)$ & $0.3427(2)$ & $0.053(2)$ & $0.039(1)$ & $0.054(2)$ & $-0.000(1)$ & $0.010(1)$ & $-0.001(1)$ \\
\hline$O(3)$ & $4 e$ & $0.5315(2)$ & $0.5769(3)$ & $0.3688(2)$ & $0.078(2)$ & $0.050(1)$ & $0.080(2)$ & $0.015(1)$ & $0.006(1)$ & $-0.013(1)$ \\
\hline$C(4)$ & $4 e$ & $0.6660(3)$ & $0.4062(3)$ & $0.4055(2)$ & $0.053(2)$ & $0.047(2)$ & $0.045(1)$ & $-0.006(1)$ & $0.004(1)$ & $-0.003(1)$ \\
\hline $\mathrm{C}(5)$ & $4 e$ & $0.7210(2)$ & $0.2942(3)$ & $0.3746(2)$ & $0.045(2)$ & $0.045(2)$ & $0.053(2)$ & $-0.005(1)$ & $0.003(1)$ & $0.007(1)$ \\
\hline$C(6)$ & $4 e$ & $0.6997(3)$ & $0.2239(4)$ & $0.2718(3)$ & $0.048(2)$ & $0.048(2)$ & $0.066(2)$ & $0.000(\mathrm{I})$ & $0.011(2)$ & $-0.010(2)$ \\
\hline$O(6)$ & $4 e$ & $0.7568(2)$ & $0.1300(4)$ & $0.2451(3)$ & $0.068(2)$ & $0.095(2)$ & $0.113(2)$ & $0.027(2)$ & $-0.001(2)$ & $-0.043(2)$ \\
\hline
\end{tabular}

* Correspondence author (e-mail: karpet@vsibml.mpi-stuttgart.mpg.de) 
Table 3. Continued.

\begin{tabular}{|c|c|c|c|c|c|c|c|c|c|c|}
\hline Atom & Site & $x$ & $y$ & $z$ & $U_{11}$ & $U_{22}$ & $U_{33}$ & $U_{12}$ & $U_{13}$ & $U_{23}$ \\
\hline $\mathrm{C}(7)$ & $4 e$ & $0.5139(3)$ & $0.1556(3)$ & $0.1943(2)$ & $0.055(2)$ & $0.040(1)$ & $0.046(2)$ & $-0.002(1)$ & $-0.000(1)$ & $-0.001(1)$ \\
\hline$C(8)$ & $4 e$ & $0.5427(3)$ & $-0.0015(3)$ & $0.2110(3)$ & $0.067(2)$ & $0.039(2)$ & $0.077(2)$ & $0.004(2)$ & $0.014(2)$ & $-0.004(2)$ \\
\hline $\mathrm{C}(9)$ & $4 e$ & $0.4500(4)$ & $-0.1040(4)$ & $0.1888(4)$ & $0.094(3)$ & $0.045(2)$ & $0.102(3)$ & $-0.014(2)$ & $0.012(3)$ & $-0.016(2)$ \\
\hline$C(10)$ & $4 e$ & $0.3537(4)$ & $-0.0738(5)$ & $0.2482(4)$ & $0.082(3)$ & $0.081(3)$ & $0.094(3)$ & $-0.036(2)$ & $-0.003(2)$ & $-0.002(3)$ \\
\hline$C(11)$ & $4 e$ & $0.3750(4)$ & $-0.0656(5)$ & $0.3654(3)$ & $0.079(3)$ & $0.081(3)$ & $0.092(3)$ & $-0.030(2)$ & $0.004(2)$ & $0.023(2)$ \\
\hline$C(12)$ & $4 e$ & $0.3739(3)$ & $0.0876(4)$ & $0.4122(3)$ & $0.071(2)$ & $0.073(3)$ & $0.067(2)$ & $-0.013(2)$ & $0.020(2)$ & $0.009(2)$ \\
\hline$C(13)$ & $4 e$ & $0.4645(3)$ & $0.1887(3)$ & $0.3887(3)$ & $0.059(2)$ & $0.050(2)$ & $0.052(2)$ & $-0.001(2)$ & $0.008(1)$ & $0.000(1)$ \\
\hline$C(14)$ & $4 e$ & $0.4574(2)$ & $0.2438(3)$ & $0.2760(2)$ & $0.042(2)$ & $0.038(1)$ & $0.056(2)$ & $0.003(1)$ & $0.004(1)$ & $0.002(1)$ \\
\hline
\end{tabular}

\section{References}

1. Braun, M.: Photochemische Cycloadditionen von Chloranil und anderen Carbonylverbindungen an Benzvalen, Homobenzvalen, Norbornadien, Norbonen und monocyclische Monoolefine. Dissertation, Universität Würzburg, Germany 1990.
2. Wolff, S.: Unpublished results.

3. Sheldrick, G. M.: Program Package SHELXTL-plus. Release 4.1. Siemens Analytical X-Ray Instruments Inc., Madison (WI 53719), USA 1990. 